\title{
Experimental Evidence of Momentum Transport Induced by an Up-Down Asymmetric Magnetic Equilibrium in Toroidal Plasmas
}

\author{
Y. Camenen, ${ }^{1}$ A. Bortolon, ${ }^{2}$ B. P. Duval, ${ }^{2}$ L. Federspiel, ${ }^{2}$ A. G. Peeters, ${ }^{1}$ F. J. Casson, ${ }^{1}$ W. A. Hornsby, ${ }^{1}$ A. N. Karpushov, ${ }^{2}$ \\ F. Piras, ${ }^{2}$ O. Sauter, ${ }^{2}$ A. P. Snodin, ${ }^{1}$ and G. Szepesi ${ }^{1}$ \\ ${ }^{1}$ Centre for Fusion, Space and Astrophysics, Department of Physics, University of Warwick, Coventry CV4 7AL, United Kingdom \\ ${ }^{2}$ Ecole Polytechnique Fédérale de Lausanne (EPFL), Centre de Recherches en Physique des Plasmas, \\ Association Euratom-Confédération Suisse, CH-1015 Lausanne, Switzerland
}

(Received 18 June 2010; published 22 September 2010)

\begin{abstract}
The first experimental evidence of parallel momentum transport generated by the up-down asymmetry of a toroidal plasma is reported. The experiments, conducted in the Tokamak à Configuration Variable, were motivated by the recent theoretical discovery of ion-scale turbulent momentum transport induced by an up-down asymmetry in the magnetic equilibrium. The toroidal rotation gradient is observed to depend on the asymmetry in the outer part of the plasma leading to a variation of the central rotation by a factor of 1.5-2. The direction of the effect and its magnitude are in agreement with theoretical predictions for the eight possible combinations of plasma asymmetry, current, and magnetic field.
\end{abstract}

DOI: 10.1103/PhysRevLett.105.135003

The fundamental issue of turbulence in magnetized plasmas is closely linked to the transport of momentum. Even in the absence of external sources, as soon as momentum is transported, steady sheared flows can develop and stabilize the turbulence by decorrelation of the eddy structures. Sheared flows can themselves enhance the transport of momentum, creating positive feedback that potentially leads to confinement bifurcations. This is particularly important in magnetic fusion devices where improved confinement regimes are desirable. In toroidal plasmas, turbulence stabilization by sheared flows arises from the $E \times B$ drift associated with a radial electric field $[1,2]$. To lowest order in $\rho_{*}$ (ratio of the ion Larmor radius to the plasma minor radius), the flow (fluid velocity) of an axisymmetric collisional plasma is purely toroidal, so the radial electric field is proportional to the parallel (magnetic field direction) flow [3]. The close relationship between the radial gradient of the parallel flow and $E \times B$ shearing rate, with the subsequent impact on turbulence, provides strong motivation for the investigation of parallel momentum transport in tokamaks. Experimentally, momentum transport is observed to exceed that expected from collisional processes (neoclassical transport) and be of the same magnitude as ion heat transport, in agreement with transport levels expected from turbulent processes [4,5]. Moreover, a peaked toroidal rotation profile can develop in the absence of momentum sources [6,7]. This observation, usually referred to as "spontaneous" or "intrinsic" rotation, demonstrates that the radial momentum flux is not only proportional to the toroidal rotation gradient but must have nondiagonal components. Importantly, intrinsic rotation is anticipated to be a key process in the International Thermonuclear Energy Reactor, which will have negligible external momentum input to the plasma core. More than two decades of research have resulted in an increasingly complete picture of parallel momentum transport by small-
PACS numbers: 52.55.Fa, 52.25.Fi, 52.30.Gz, 52.35.Ra

scale (ion Larmor radius) turbulence. The proposed mechanisms all involve a breaking of the symmetry along magnetic field lines, as otherwise the radial flux of momentum above and below the plasma horizontal midplane exactly compensates, resulting in a zero net transport across a flux surface [8]. Among the recent theoretical findings, symmetry breaking arising from a shaped magnetic equilibrium was shown to generate a residual stress (a turbulent momentum flux proportional neither to the toroidal rotation nor to its radial gradient) with a magnitude large enough to modify the intrinsic rotation [9]. The effect relies on the shift of the turbulence maximum away from the low field side midplane, which leads to a partial compensation of the momentum flux in the upper and lower parts of the plasma (see Fig. 1 in Ref. [10]), thus generating a net momentum flux. The sign of the flux (inward or outward) naturally changes if the plasma asymmetry is reversed but also with the toroidal magnetic field and plasma current direction, a consequence of the system invariance under parity transformations. This Letter reports the first experimental test of the up-down asymmetry residual stress prediction, and full agreement is demonstrated. The experiments build on the link between residual stress and the intrinsic rotation gradient. Indeed, in an axisymmetric device, toroidal angular momentum conservation implies that the flux surface averaged radial flux of toroidal angular momentum cancels when stationary conditions are reached in the absence of a momentum source. Then, by approximating the parallel and toroidal directions, assuming that the momentum flux is turbulent, and using a decomposition according to the considered symmetry breaking mechanisms (no particle flux), the toroidal rotation gradient $v_{\phi}^{\prime}=-\partial v_{\phi} / \partial r$ may be written:

$$
v_{\phi}^{\prime}=-[\underbrace{R V_{\mathrm{co}} v_{\phi}}_{\text {pinch }}+\underbrace{C_{E \times B}+C_{\mathrm{FS}}+C_{\rho_{*}}}_{\text {residual stress }}] / \chi_{\|},
$$


with $r=\left(R_{\max }-R_{\min }\right) / 2$ a flux surface label (radial coordinate). The parallel momentum diffusivity $\chi_{\|}$comes from the diagonal contribution of the momentum flux that tends to relax the rotation gradient [4]. The Coriolis pinch [11] is proportional to the background rotation and usually enhances the rotation gradient. Residual stress can arise from symmetry breaking by $E \times B$ shearing $C_{E \times B}$ [12], up-down asymmetric flux surfaces $C_{\mathrm{FS}}$ [9], or $\rho_{*}$ effects $C_{\rho_{*}}[13,14]$. As emphasized in Eq. (1), a change in the sign of the up-down asymmetry flux $C_{\mathrm{FS}}$ will change the, experimentally observable, toroidal rotation gradient $v_{\phi}^{\prime}$.

Experiments were performed on the Tokamak à Configuration Variable (TCV) [15] in deuterium plasmas with large up-down asymmetry (Fig. 1). A total of eight magnetic configurations are considered covering all combinations of plasma shape $s^{+/-}$, magnetic field $b^{+/-}$, and plasma current $j^{+/-}$directions that all change the sign of the predicted up-down asymmetry flux. $s^{+}$is used to label the plasma shape with positive top triangularity (left plot in Fig. 1). The toroidal magnetic field $b$, plasma current $j$, and toroidal rotation $v_{\phi}$ are defined positive in the clockwise direction viewed from above. We first focus on the $b^{-} j^{+}$ configuration and consider a change of the plasma shape $s^{+/-}$. The asymmetric configurations are compared while keeping the other parameters as similar as possible. The plasma current is $I_{p}=340 \pm 3 \mathrm{kA}$, and the magnetic field $B=1.4 \pm 0.02 \mathrm{~T}$ corresponding to a safety factor of $q_{95}=2.9 \pm 0.05$ at $95 \%$ of the poloidal flux. The line averaged plasma density is maintained at $\bar{n}_{e}=$ $4.1 \pm 0.1 \times 10^{19} \mathrm{~m}^{-3}$ and the kinetic profiles collected
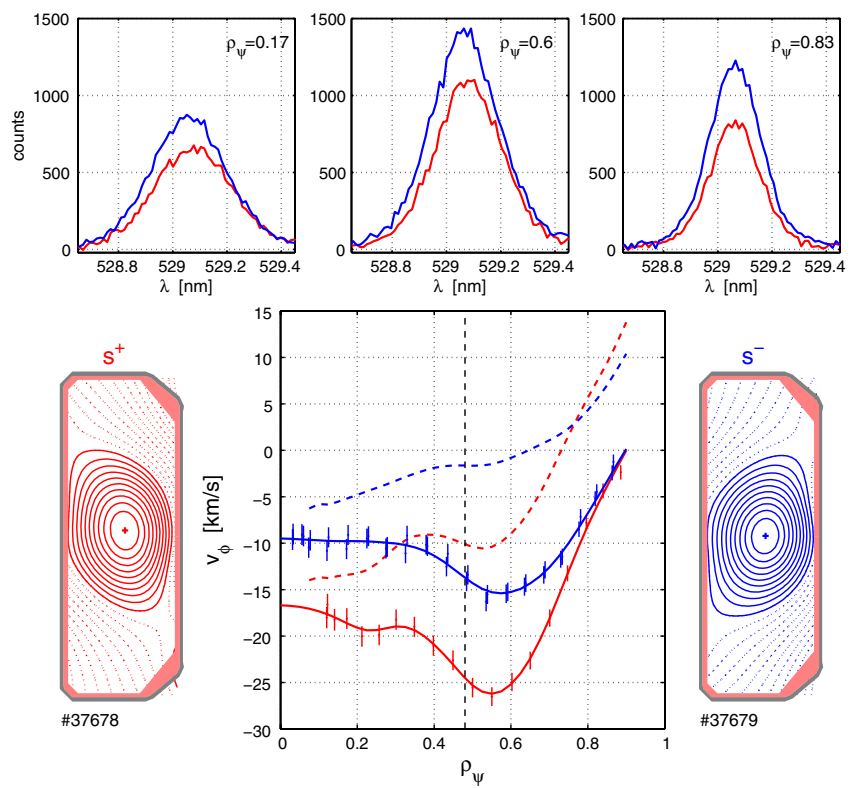

FIG. 1 (color online). CXRS spectra measured at three radial location (top plots) for the $s^{+}$(red) and $s^{-}$(blue) configurations $\left(b^{-} j^{+}\right)$shown in the bottom left and right plots (poloidal flux contours). The toroidal rotation profiles of the carbon impurity (full line) and main ions (dashed line) are shown in the middle bottom plot. Positive values: clockwise viewed from above. over $450 \mathrm{~ms}$ (about 12 energy confinement times) once stationary conditions are reached. The electron temperature and density profiles measured by using Thomson scattering and the $\mathrm{C}^{6+}$ temperature and density profiles from charge exchange recombination spectroscopy (CXRS) [16] are shown in Fig. 2 as a function of the square root of the normalized poloidal flux $\rho_{\psi}$, including the measurement error bars and a cubic spline fit with tension. Within the experimental uncertainties, the temperature and density profiles are the same for both magnetic configurations with the exception of the carbon density, which is $\sim 20 \%$ higher in the $s^{-}$configuration. The toroidal rotation is measured from the Doppler shift of the carbon VI $(n=$ $8 \rightarrow 7,529.1 \mathrm{~nm}$ ) radiation resulting from collisions of carbon impurities with hydrogen neutrals from a diagnostic neutral beam (DNBI, $E \approx 50 \mathrm{keV}, I=3 \mathrm{~A}$ ) [17]. Quasiperpendicular injection, low power, and high beam energy result in a negligible DNBI induced rotation $(<2 \mathrm{~km} / \mathrm{s})$, while CXRS provides local measurements of the carbon rotation with a radial resolution $\Delta \rho_{\psi}<0.06(\Delta r<$ $1.4 \mathrm{~cm}$ ) and less than $2 \mathrm{~km} / \mathrm{s}$ uncertainties including wavelength calibration and statistical errors. Toroidal rotation profiles of the $\mathrm{C}^{6+}$ impurity are shown in Fig. 1 together with examples of the DNBI induced CXRS spectra. The sawtooth inversion radius, measured by using a multiwire chamber soft $\mathrm{x}$-ray camera [18] (spatial resolution $\Delta \rho_{\psi}<0.05$ at the midplane), is also indicated by a vertical dashed line in Figs. 1 and 2. In both configurations, the plasma core rotates in the countercurrent direction, and the rotation profile is strongly flattened (possibly slightly hollow) inside the sawtooth inversion radius [19] as are the ion and electron pressure profiles. Outside the inversion radius, $v_{\phi}^{\prime}$ is negative and 1.5-2 times larger (in absolute

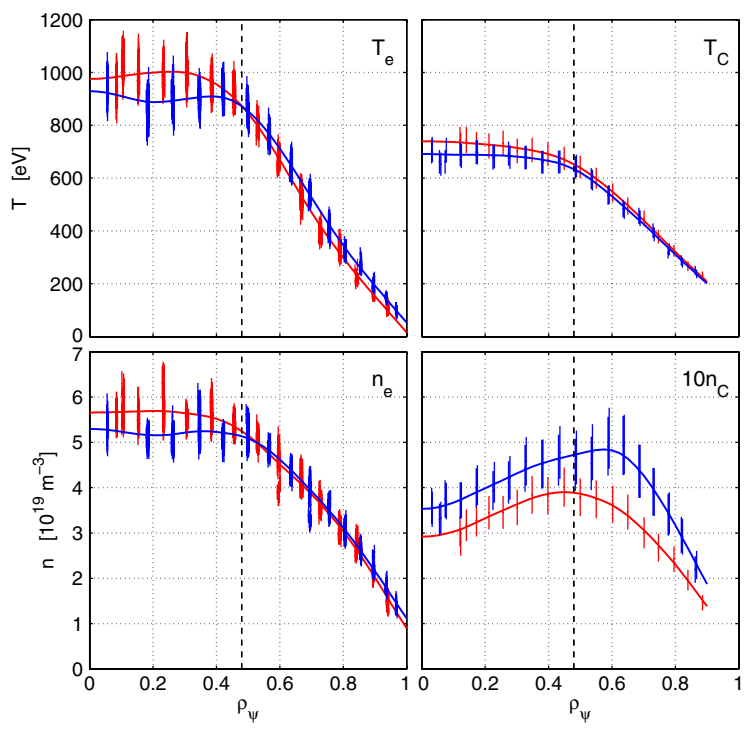

FIG. 2 (color online). Left: Electron temperature (top) and density (bottom) from Thomson scattering. Right: Carbon temperature (top) and density (bottom) from CXRS. The profiles are shown for the $s^{+}$(red) and $s^{-}$(blue) cases of Fig. 1. The vertical dashed line indicates the sawtooth inversion radius. 
TABLE I. Deuterium (large font size) and carbon (small font size) values of $\Delta v_{\phi}$ in $\mathrm{km} / \mathrm{s}$ for the various configurations. Cocurrent central rotation cases are indicated by an asterisk. $s_{s} s_{b} s_{j} \operatorname{sgn}\left(\Delta v_{\phi}\right)>0$ cases are highlighted in bold characters.

\begin{tabular}{|c|c|c|c|c|c|c|c|c|}
\hline$b^{+}$ & \multicolumn{2}{|c|}{$j^{+}$} & \multicolumn{2}{|l|}{$j^{-}$} & $b^{-}$ & \multicolumn{2}{|c|}{$j^{+}$} & $j^{-}$ \\
\hline$s^{+}$ & -10.1 & -12.4 & $\cdots$ & & $s^{+}$ & -15.8 & -18.6 & 18.7 \\
\hline & $-1.1^{*}$ & -1.3 & $-5.9^{*}$ & -4.9 & & . & & $\ldots$ \\
\hline$s^{-}$ & -18.0 & -19.4 & $\cdots$ & & $s^{-}$ & -6.9 & -11 & 5.8 \\
\hline & $\cdots$ & & $-1.9^{*}$ & -0.1 & & $-1.1^{*}$ & -3.4 & $\cdot$ \\
\hline
\end{tabular}

terms) for $s^{+}$than $s^{-}$, leading to a similar difference in the central toroidal rotation achieved at matched edge rotation values. This observation is in agreement with the predicted effect of the up-down asymmetry flux $C_{\mathrm{FS}}$ that is positive for $s^{+}$and negative for $s^{-}$(with $b^{-} j^{+}$). According to Eq. (1), this translates into a more negative value of $v_{\phi}^{\prime}$ for $s^{+}$than $s^{-}$. For an up-down symmetric case, the toroidal rotation profile lies between the ones of the $s^{+/-}$ cases, in agreement with the prediction that $C_{\mathrm{FS}}=0$. As Eq. (1) describes the bulk ions (deuterium), it is important to check that the neoclassical difference between carbon and deuterium toroidal rotation do not affect these results. The deuterium toroidal rotation, calculated by using the neoclassical transport code NEOART [20], is found significantly more cocurrent than the carbon rotation (Fig. 1), as expected in a medium-size device like TCV where the diamagnetic velocity is relatively large. The change in $v_{\phi}^{\prime}$ between the $s^{+/-}$configurations is, however, approximately similar for carbon and deuterium, so the modeled neoclassical correction does not affect the interpretation.

As mentioned in the introduction, the sign of $C_{\mathrm{FS}}$ depends not only on the shape orientation but also on the direction of the magnetic field and plasma current, enhancing positive toroidal rotation gradient $v_{\phi}^{\prime}$ when $s_{s} s_{b} s_{j}>0$. To discriminate between the effect of the up-down asymmetry flux and other possible effects that do not obey the same transformation rules, the comparison is therefore extended to all the $s^{+/-} b^{+/-} j^{+/-}$configurations. It allows us, for instance, to allay any effect of top-bottom or toroidal asymmetries in the vessel or coils resulting from con- struction inaccuracies. In Table I, $\Delta v_{\phi}=\left.v_{\phi}\right|_{\rho_{\psi}=0.65}-$ $\left.v_{\phi}\right|_{\rho_{\psi}=0.85}$ is used to quantify the variation of the intrinsic rotation profile for the deuterium and carbon species. It provides an estimate of the average toroidal rotation gradient $\bar{v}_{\phi}^{\prime}$ in the outer part of the plasma. The range $\Delta \rho_{\psi}=$ $0.65-0.85$ corresponds to a variation in the minor radius of $\Delta r=-5.55 \mathrm{~cm}$ with a difference of less than $5 \%$ between the configurations. It covers a region from well outside the sawtooth inversion radius to just inside the last CXRS measurement points. While the core of TCV $L$-mode plasmas usually rotates in the countercurrent direction, it can also rotate in the cocurrent direction $[19,21,22]$. Such a reversed rotation state is, quite interestingly, sometimes observed in the present experiments. We first discuss the cases with countercurrent rotation (first line in Table I). In all the configurations where $s_{s} s_{b} s_{j}>0, \bar{v}_{\phi}^{\prime}$ is more positive than for those with $s_{s} s_{b} s_{j}<0$, consistently with the change of sign of $C_{\mathrm{FS}}$, leading to larger values of $\left|\bar{v}_{\phi}^{\prime}\right|$ when $s_{s} s_{b} s_{j} \operatorname{sgn}\left(\bar{v}_{\phi}^{\prime}\right)>0$. For the reversed rotation cases (second line in Table I), the toroidal rotation profile is no longer monotonic and exhibits an extremum between $\rho_{\psi}=0.65$ and 0.85 . Smaller values of $\left|\bar{v}_{\phi}^{\prime}\right|$ are therefore obtained in comparison with the nonreversed cases. However, the highest values of $\left|\bar{v}_{\phi}^{\prime}\right|$ are still obtained when $s_{s} s_{b} s_{j} \operatorname{sgn}\left(\bar{v}_{\phi}^{\prime}\right)>0$. Thus, although the intrinsic rotation profile results from the interplay of mechanisms that are not all completely understood, the differential effect of the up-down asymmetry is a robust observation that is independent of the initial rotation profile. This up-down asymmetry effect is observed in plasmas with similar pressure profiles and global parameters, comparable MHD levels, no external momentum input, and a negligible magnetic field ripple effect. From previous studies, scrape-off layer (SOL) flows depend on the position of the $X$ point $[23,24]$ or plasma wall contact point [25] and can affect the rotation profile by changing the boundary condition. In the present experiments, the effect of the updown asymmetry on the toroidal rotation gradient is still observed when the rotation at $\rho \sim 0.85$ is matched (see Fig. 1), which precludes SOL flows being responsible for the change in $\bar{v}_{\phi}^{\prime}$. Some of the cases in Table I, however,

TABLE II. Parameters used in the linear simulations. The density, temperature, and normalized gradients are indicated by $n, T$, and $R / L_{X}=-R_{0} \partial X / \partial r$, respectively, with the subscripts $e, i$, and $C$ indicating the electron, deuterium, and carbon species, respectively ( $T_{i}$ and $T_{C}$ are assumed equal). The normalized deuterium rotation $\hat{u}_{i}$ and its gradient $\hat{u}_{i}^{\prime}$ are also indicated.

\begin{tabular}{|c|c|c|c|c|c|c|c|c|c|c|}
\hline$s^{+}$ & $\frac{R}{L_{T_{i}}}$ & $\frac{R}{L_{T_{e}}}$ & $\frac{R}{L_{n_{i}}}$ & $\frac{R}{L_{n_{e}}}$ & $\frac{R}{L_{n_{C}}}$ & $\frac{n_{i}}{n_{e}}$ & $\frac{n_{C}}{n_{e}}$ & $\frac{T_{e}}{T_{i}}$ & $\hat{u}_{i}^{\prime}$ & $\hat{u}_{i}$ \\
\hline$\rho_{\psi_{1}}$ & 7.1 & 10.6 & 6.6 & 5.7 & 4.8 & 0.52 & 0.08 & 1.1 & -0.24 & 0.03 \\
\hline$\rho_{\psi_{2}}$ & 9.1 & 14.2 & 8.0 & 8.0 & 8.0 & 0.52 & 0.08 & 1.0 & -0.34 & -0.01 \\
\hline$\rho_{\psi_{3}}$ & 11.2 & 21.0 & 10.8 & 11.8 & 13.2 & 0.58 & 0.07 & 0.82 & -0.35 & -0.05 \\
\hline$s^{-}$ & $\frac{R}{L_{T_{i}}}$ & $\frac{R}{L_{T_{e}}}$ & $\frac{R}{L_{n_{i}}}$ & $\frac{R}{L_{n_{e}}}$ & $\frac{R}{L_{n_{C}}}$ & $\frac{n_{i}}{n_{e}}$ & $\frac{n_{C}}{n_{e}}$ & $\frac{T_{e}}{T_{i}}$ & $\hat{u}_{i}^{\prime}$ & $\hat{u}_{i}$ \\
\hline$\rho_{\psi_{1}}$ & 7.0 & 8.6 & 6.7 & 4.9 & 3.7 & 0.4 & 0.1 & 1.3 & -0.07 & 0.0 \\
\hline$\rho_{\psi_{2}}$ & 9.0 & 11.8 & 7.0 & 7.0 & 7.0 & 0.4 & 0.1 & 1.17 & -0.13 & -0.01 \\
\hline$\rho_{\psi_{3}}$ & 11.5 & 16.8 & 7.3 & 10.0 & 12.2 & 0.46 & 0.09 & 1.0 & -0.27 & -0.04 \\
\hline
\end{tabular}



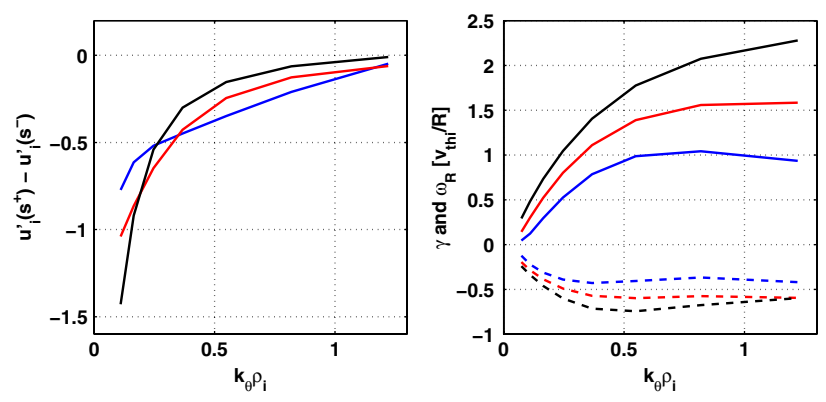

FIG. 3 (color online). Left: Variation of the intrinsic rotation gradient $\hat{u}_{i}^{\prime}\left(s^{+}\right)-\hat{u}_{i}^{\prime}\left(s^{-}\right)$of deuterium assessed from linear GKW simulations for $\rho_{\psi_{1}}$ (blue), $\rho_{\psi_{2}}$ (red), and $\rho_{\psi_{3}}$ (black) as a function of the wave vector $k_{\theta} \rho_{i}$. Right: Corresponding mode growth rate $\gamma$ (full line) and real frequency $\omega_{R}$ (dashed line), negative values meaning in the electron diamagnetic drift direction.

show a difference in the edge rotation that could be induced by SOL flows. This change in the boundary conditions could be responsible for some of the scatter in Table I, although it cannot account for the systematic variation of $\bar{v}_{\phi}^{\prime}$ reported here. For these conditions, the only mechanism which, to our knowledge, can reproduce the change of $\bar{v}_{\phi}^{\prime}$ with $s_{s}, s_{b}$, and $s_{j}$, for co- and countercurrent rotation, is a modification of the turbulent momentum transport induced by the up-down asymmetry $[9,10]$.

We now examine the magnitude of the up-down asymmetry flux $C_{\mathrm{FS}}$. Linear calculations of the turbulent momentum flux were performed with the gyrokinetic fluxtube code GKW $[26,27]$ at three radii, $\rho_{\psi}=0.65,0.75$, and 0.85 , for the plasmas described in Figs. 1 and 2 (simulation parameters in Table II). Three kinetic species (electron, deuterium, and carbon) were included with electrostatic fluctuations considered in the collisionless limit. The effect of $E \times B$ shearing and $\rho_{*}$ effects (local approximation) are neglected. Note that when the $E \times B$ shearing rate is proportional to the rotation gradient, it enters only the diagonal momentum flux $[28,29]$. The gyrokinetic equation solved in GKW is formulated in the frame rotating with the toroidal angular velocity of the plasma $\omega_{\phi}=v_{\phi} / R$ at the flux surface under investigation [30]. For each case, three simulations are performed with different values of the normalized rotation $\hat{u}=s_{b} \frac{R_{0} \omega_{\phi}}{v_{\text {thi }}}$ and of its gradient $\hat{u}^{\prime}=$ $-s_{b} \frac{R_{0}^{2}}{v_{\text {thi }}} \frac{B_{t}}{B} \frac{\partial \omega_{\phi}}{\partial r}$, with $R_{0}$ the magnetic axis major radius and $v_{\text {thi }}=\sqrt{2 T_{i} / m_{i}}$ the main ion thermal velocity, to decompose the momentum flux into a diagonal $\chi_{\|}$, pinch $R V_{\text {co }}$ and residual stress $C_{\mathrm{FS}}$ parts, as in Eq. (1). These coefficients are then used to compute $\hat{u}_{i}^{\prime}\left(s^{+}\right)-\hat{u}_{i}^{\prime}\left(s^{-}\right)$. The linear results depend strongly on the mode wave vector $k_{\theta} \rho_{i}$ with a larger effect of the up-down asymmetry for lower wave vectors (Fig. 3). The overall momentum flux will be the weighted average of each wave vector contribution resulting from the nonlinear interactions. Following [31], a quasilinear estimate is obtained from a mixing length rule, leading to $\hat{u}_{i}^{\prime}\left(s^{+}\right)-\hat{u}_{i}^{\prime}\left(s^{-}\right)=-0.42,-0.44$, and -0.4 , to be compared to the experimental values -0.17 , -0.21 , and -0.08 for $\rho_{\psi}=0.65,0.75$, and 0.85 , respectively. A nonlinear study including the effect of collisions and $E \times B$ shearing will be addressed in a future work for a full quantitative comparison. In summary, an up-down asymmetry effect on the intrinsic rotation profile was first predicted and has now been demonstrated experimentally. Preliminary simulations provide the correct order of magnitude change in the rotation profiles. These experiments evidence the importance of turbulent momentum transport in intrinsic rotation, in particular, the role of off-diagonal contributions, stressing that effects like the up-down asymmetry flux need to be considered to predict intrinsic rotation in magnetic fusion devices.

[1] T. Chiueh et al., Phys. Fluids 29, 231 (1986).

[2] K. H. Burrell et al., Phys. Plasmas 1, 1536 (1994).

[3] F. L. Hinton et al., Phys. Fluids 28, 3082 (1985).

[4] N. Mattor et al., Phys. Fluids 31, 1180 (1988).

[5] S. D. Scott et al., Phys. Rev. Lett. 64, 531 (1990).

[6] L-G. Eriksson et al., Plasma Phys. Controlled Fusion 39, 27 (1997).

[7] J.E. Rice et al., Nucl. Fusion 47, 1618 (2007).

[8] A. G. Peeters et al., Phys. Plasmas 12, 072515 (2005).

[9] Y. Camenen et al., Phys. Rev. Lett. 102, 125001 (2009).

[10] Y. Camenen et al., Phys. Plasmas 16, 062501 (2009).

[11] A. G. Peeters, C. Angioni, and D. Strintzi, Phys. Rev. Lett. 98, 265003 (2007).

[12] R. R. Dominguez et al., Phys. Fluids B 5, 3876 (1993).

[13] A. G. Peeters et al., Plasma Phys. Controlled Fusion 48, B413 (2006).

[14] C. J. McDevitt et al., Phys. Plasmas 16, 052302 (2009).

[15] F. Hofmann et al., Plasma Phys. Controlled Fusion 36, B277 (1994).

[16] A. Bortolon, EPFL thesis 4569, 2009.

[17] A. N. Karpushov et al., Fusion Eng. Des. 66-68, 899 (2003).

[18] A. V. Sushkov et al., Rev. Sci. Instrum. 79, 10E319 (2008).

[19] B. P. Duval et al., Plasma Phys. Controlled Fusion 49, B195 (2007).

[20] A. G. Peeters et al., Phys. Plasmas 7, 268 (2000).

[21] A. Bortolon et al., Phys. Rev. Lett. 97, 235003 (2006).

[22] B. P. Duval et al., Phys. Plasmas 15, 056113 (2008).

[23] B. LaBombard et al., Phys. Plasmas 15, 056106 (2008).

[24] J.E. Rice et al., Plasma Phys. Controlled Fusion 50, 124042 (2008).

[25] J. Gunn et al., J. Nucl. Mater. 363-365, 484 (2007).

[26] A. G. Peeters et al., Phys. Plasmas 11, 3748 (2004).

[27] A. G. Peeters et al., Comput. Phys. Commun. 180, 2650 (2009).

[28] R. E. Waltz et al., Phys. Plasmas 14, 122507 (2007).

[29] F. J. Casson et al., Phys. Plasmas 16, 092303 (2009).

[30] A. G. Peeters et al., Phys. Plasmas 16, 042310 (2009).

[31] E. Fable et al., Plasma Phys. Controlled Fusion 52, 015007 (2010). 